Research Paper

\title{
The Significance of iron deficiency and anemia in a real-life COPD cohort
}

\author{
Alex Pizzini ${ }^{*}$, Magdalena Aichner ${ }^{1^{*}}$, Thomas Sonnweber ${ }^{1}$, Ivan Tancevski1 ${ }^{1}$, Günter Weiss ${ }^{1,2}{ }^{\bowtie}$ and Judith \\ Löffler-Ragg1 ${ }^{\bowtie}$ \\ 1. Department of Internal Medicine II, Infectious Diseases, Pneumology, Rheumatology, Medical University of Innsbruck, Innsbruck, Austria. \\ 2. Christian Doppler Laboratory for Iron Metabolism and Anemia Research, Medical University of Innsbruck, Austria. \\ *These authors contributed equally to this manuscript. \\ $\square$ Corresponding authors: Judith Löffler-Ragg and Günter Weiss, E-mail: judith.loeffler@i-med.ac.at (J.L.); guenter.weiss@i-med.ac.at (G.W). Medical \\ University of Innsbruck, Department of Internal Medicine II, Anichstraße 35, A-6020, Innsbruck, Austria. Phone: + 43 (0) 512-504-23251; Fax: +43 (0) \\ 512-504-23317. \\ () The author(s). This is an open access article distributed under the terms of the Creative Commons Attribution License (https://creativecommons.org/licenses/by/4.0/). \\ See http://ivyspring.com/terms for full terms and conditions.
}

Received: 2020.03.19; Accepted: 2020.07.12; Published: 2020.08.19

\begin{abstract}
Background: Current evidence suggests an increased prevalence of iron deficiency (ID) and anemia in chronic obstructive pulmonary disease (COPD). ID and subsequent anemia can be due to iron losses via bleeding resulting in absolute ID or inflammation-driven retention of iron within macrophages resulting in functional ID and anemia of inflammation.

Methods: This is a retrospective analysis of 204 non-exacerbated COPD patients in outpatient care. Current definitions of absolute and functional ID were applied to determine the prevalence of ID and to analyze associations to disease severity in terms of lung function parameters and clinical symptoms.

Results: The studied cohort of COPD patients demonstrated a high prevalence of ID, ranging from 30 to $40 \%$ during the observation time. At the initial presentation, absolute or functional ID was found in $9.3 \%$ to $12.3 \%$ of COPD individuals, whereas combined forms of absolute and functional ID were most prevalent ( $25.9 \%$ of all individuals). The prevalence of ID increased during longitudinal follow-up ( $37 \pm 15$ months), and especially combined forms of ID were significantly related to anemia. Anemia prevalence ranged between $14.2 \%$ and $20.8 \%$ during the observation period and anemia was associated with lower FEVI, DLCOc, and CRP elevation. Accordingly, ID was associated with decreased FEV1, DLCOc, and an elevation in CRP.

Conclusion: ID is common in COPD patients, but a uniform definition for accurate diagnosis does not exist. Prevalence of functional ID and anemia increased during follow-up. The associations of ID and anemia with reduced functional lung capacity and elevated inflammation may reflect a more severe COPD phenotype.
\end{abstract}

Key words: COPD, obstructive pulmonary disease, anemia, iron deficiency, inflammation

\section{Introduction}

Chronic obstructive pulmonary disease (COPD) is the third leading cause of death worldwide, resulting in 3.0 million deaths in 2016 [1]. The main pathogenetic features of COPD are oxidative stress and protease/antiprotease imbalance leading to local airway inflammation and remodeling as well as chronic systemic inflammation [2-4].

Iron is a crucial micronutrient as it is involved in several metabolic processes, such as DNA synthesis, oxygen transport, cellular metabolism, and mitochondrial respiration $[5,6]$. On the other hand, environmental sources of iron and other particles can disrupt and interfere with local iron-homeostasis in the lung [7]. Genes related to iron-metabolism are associated with COPD, and exposure to tobacco smoking, air pollution, and other harmful substances impact on regulatory mechanisms, potentially driving the pathogenesis of COPD [7-9]. However, these 
processes are not limited to the lung, as inflammatory cytokines that are induced and released in the course of COPD [10, 11] can also impact iron homeostasis. Thereby, inflammatory cytokines and the increased expression of the master regulator of iron homeostasis hepcidin result in increased acquisition and storage of iron within cells of the reticuloendothelial system. This results in functional iron deficiency (FID) and anemia of inflammation (AI), reflected by low circulating iron levels and thus reduced availability of the metal for erythropoietic cells, whereas levels of the iron storage protein ferritin are normal or increased as a consequence of reticuloendothelial iron retention [11].

In contrast, true or absolute iron deficiency (AID) and subsequent iron deficiency anemia (IDA) occurs as a consequence of chronic blood losses and/or insufficient dietary iron absorption [12]. Thus, low circulating iron and ferritin levels characterize this most frequent type of anemia. Besides, combined forms of AI and IDA (CID) can occur in patients with inflammation-driven iron retention and chronic gastrointestinal or urogenital blood losses [13]. The identification of such patients is challenging because no single parameter can differentiate between $\mathrm{AI}$ and AI with combined IDA but of significant clinical interest. Patients with IDA and patients with combined AI/IDA benefit from iron supplementation. In contrast, its benefit-risk assessment has not been established in prospective trials in patients with FID, as it may be hampered by ongoing inflammation [11]. Several clinical markers have been used to differentiate between AI and AI/IDA, including the soluble transferrin receptor (sTfR) reflecting the needs of iron for erythropoiesis [14-16]. As it is also affected by inflammation, a ratio of sTfR/log ferritin was introduced, which should better differentiate between AI and AI/IDA [17]. However, some overlap, especially in patients with advanced inflammation, remains $[16,18]$.

This retrospective study aimed to identify the prevalence of ID with/without concomitant anemia by applying robust and easily reproducible definitions for different types of ID and anemia in a real-life COPD cohort and to evaluate its impact on disease course.

\section{Methods}

\section{Patients selection}

This is a single-center cohort study, including 204 COPD patients based on the COPD registry of the Pulmonary Outpatient Clinic of the Department of Internal Medicine II Innsbruck. Patients who were in routine treatment at the Pulmonary Outpatient Clinic between July 2007 and May 2017 were retrospectively recruited. All COPD patients were screened and included if the diagnostic criteria according to the Global Initiative for Chronic Obstructive Lung Disease (GOLD) guidelines [19], i.e., a post-bronchodilator fixed ratio of forced expiratory volume in one second (FEV1) versus forced vital capacity (FVC) less than 0.7 and COPD-typical symptoms were met. Patients in outpatient care with no evidence of acute infection or exacerbation (AECOPD) and only low-grade inflammation (CRP < $5 \mathrm{mg} / \mathrm{dl}$ ) were eligible. Further, only patients with available laboratory blood tests, including iron parameters on the day of lung function testing were included. Follow-up lung function tests and laboratory parameters were admitted if available from at least 24 months later. Patients were followed up as part of routine clinical management at the institution, either because they are not in treatment at an out-of-hospital licensed pulmonologist or have conditions requiring a specialized outpatient clinic. Figure 1 shows the algorithm of patient selection.

Parameters related to COPD like spirometry, diffusion capacity for carbon monoxide corrected for hemoglobin (DLCOc), COPD assessment test (CAT) results, the frequency of severe acute exacerbations requiring hospitalization, and routine laboratory parameters were included in this analysis. Gastrointestinal comorbidities and anticoagulation therapy (ACT), defined as vitamin - $\mathrm{k}$ antagonists, factor-Xa inhibitors, low-molecular-weight heparin, and platelet aggregation inhibitors were assessed.

\section{Iron deficiency and anemia definitions}

Different clinical definitions of ID were applied to the study cohort, as shown in Table 1.

Anemia was defined by hemoglobin levels below $120 \mathrm{~g} / 1$ for women and below $130 \mathrm{~g} / 1$ for men and categorized as IDA, AI, or a combination of AI and IDA, as shown in Table 2.

Table 1. ID definitions (11)

\begin{tabular}{lll}
\hline Definition & Ferritin $(\mu \mathrm{g} / \mathrm{L})$ & TSAT $(\%)$ \\
\hline Absolute ID (AID) & $\leq 30$ & $\leq 20$ \\
Functional ID (FID) & $\geq 100$ & $\leq 20$ \\
Combined ID (CID) * & $30-100$ & $\leq 20$ \\
No ID & $>30$ & $>20$ \\
*In five cases the constellation of ferritin < 30 ug/L and TSAT $>20 \%$ with Tf being \\
in the normal range was categorized as combined ID.
\end{tabular}

Table 2. Anemia definitions based on ferritin, TSAT, and CRP levels (11)

\begin{tabular}{llll}
\hline Definition & Ferritin $(\mu \mathrm{g} / \mathrm{L})$ & TSAT $(\%)$ & CRP $(\mathrm{mg} / \mathrm{dL})$ \\
\hline Iron deficiency anemia (IDA) & $\leq 30$ & & $\leq 0.5$ \\
Anemia of inflammation (AI) & $\geq 100$ & $\leq 20$ & $>0.5$ \\
Combined (CID = AI + IDA) & $<100$ & & $>0.5$ \\
\hline
\end{tabular}




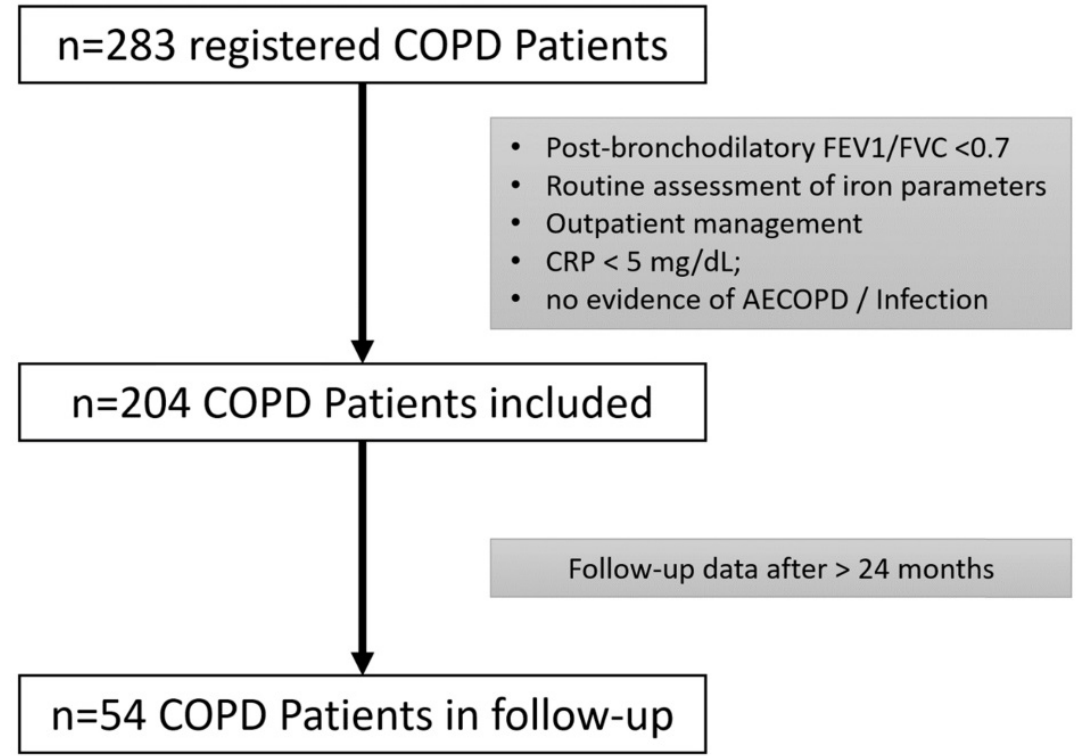

Figure 1. Algorithm of patient selection.

\section{Statistical analysis}

Mean comparison of normally distributed numeric data was performed using Student's $t$-test. If Gaussian distribution was not given, the MannWhitney-U-test and Kruskal-Wallis-test were applied. Baseline characteristics in terms of categorical variables were compared using Chi-Square, and Fisher's exact test, where appropriate. Spearman rank correlation technique was used for analysis of monotonic associations in non-normally distributed data. If Gaussian distribution based on Shapiro-Wilk test and a linear relationship were given, Pearson correlation coefficient was calculated to assess the degree of correlation. Trends over time analyses were conducted with paired Student's $t$-test, Wilcoxon test, or Nemar's test, as appropriate. All tests were two-sided and a $p$-value of less than 0.05 indicated statistical significance. A post-hoc power calculation determined a power of 0.92 for the detection of ID assuming a prevalence of ID among COPD patients of $20 \%$ according to previous data [21]. Statistical analyses were performed with the SPSS 24.0 statistical package (IBM Corp., Armonk, NY, USA).

\section{Ethical approval}

All procedures performed in the present study involving human participants were in accordance with the ethical standards of the Institutional and/or National Research Committee and with the 1964 Helsinki declaration and its later amendments and were performed after approval of the Ethics Committee of the Medical University of Innsbruck (EK Nr.1147/2017).

\section{Results}

\section{Demographics and iron-metabolism of the study cohort}

The study population included 134 (65.7\%) male and $70(34.3 \%)$ female patients, the mean age was $64.80 \pm 10.16$ years and the mean follow-up period was $36.83 \pm 14.70$ months. Patients were allocated to the four COPD stages based on their FEV1 capacity, as defined by the GOLD classification [19]. Baseline characteristics, as well as follow-up data of the study population, are shown in Table 3.

During follow-up, significant decreases in FEV1 $(p<0.01)$ and DLCOc $(p<0.01)$ over time were observed in the entire cohort. At least one AECOPD requiring in-hospital treatment was reported from 11 (22.4 \%) patients during the observation period. No significant differences in the frequency of ID or in absolute iron parameters were found when comparing patients with and without exacerbations. Table 3 shows the respective iron parameters at both time-points. Ferritin was the only iron parameter to show a significant change during follow-up with decreasing means $(p=0.02$, Table 3$)$. When looking at the correlations between FEV1 and iron parameters at study inclusion, a significant, inverse relationship could be found for sTfR $(r=-0.27, p=0.02$, Figure 2), and ferritin-index $(r=-0.23, p=0.04$, Figure 2$)$, whereas no significant correlations were seen between FEV1 and serum iron, ferritin, Tf, and TSAT. CAT-score did not correlate with iron metabolism at study inclusion, nor did FEV1 or CAT-score at follow-up. In contrast, most of the iron parameters revealed a significant correlation with DLCOc at study inclusion (serum 
iron $r=0.21, p<0.01$; Tf $r=-0.172 . p=0.03$; TSAT $r=0.246$, $p<0.01$; sTfR $r=-0.341, p<0.01$; ferritin-index $r=-0.309$, $p=0.01)$.

Table 3. Baseline characteristics of the study population, at study inclusion, and during follow-up

\begin{tabular}{|c|c|c|c|c|c|c|}
\hline Parameter & & $\mathrm{n}$ & $\begin{array}{l}\text { Study } \\
\text { inclusion }\end{array}$ & $\mathrm{n}$ & Follow-up & $p$-value \\
\hline $\begin{array}{l}\text { Follow-up } \\
\text { (months) }\end{array}$ & $36.83 \pm 14.70$ & & & & & \\
\hline Age (years) & & 204 & $64.80 \pm 10.164$ & 54 & $66.37 \pm 10.164$ & \\
\hline COPD stages\# & & 204 & & & & \\
\hline Gold 1 & & & $8(3.9 \%)$ & & & \\
\hline Gold 2 & & & $87(42.6 \%)$ & & & \\
\hline Gold 3 & & & $63(30.9 \%)$ & & & \\
\hline Gold 4 & & & $46(22.5 \%)$ & & & \\
\hline FEV1 (\%) & & 204 & $50.32 \pm 17.37$ & 50 & $48.36 \pm 19.47$ & $<0.01$ \\
\hline DLCOc & & 166 & $59.42 \pm 21.68$ & 44 & $55.25 \pm 22.80$ & $<0.01$ \\
\hline CAT Score* & & 146 & $15.34 \pm 8.11$ & 34 & $18.67 \pm 16.72$ & 0.98 \\
\hline $\mathrm{ACT}^{\#}$ & & 204 & $116(56.9 \%)$ & & & \\
\hline GI comorbidity\# & & 204 & $54(26.5 \%)$ & & & \\
\hline AECOPD $\#$ & & 49 & $11(22.4 \%)$ & & & \\
\hline $\begin{array}{l}\text { Laboratory } \\
\text { parameters }\end{array}$ & Ref. range & & & & & \\
\hline $\begin{array}{l}\text { serum iron } \\
(\mu \mathrm{mol} / \mathrm{L})\end{array}$ & $5.8-34.5$ & 204 & $16.92 \pm 7.08$ & 54 & $20.14 \pm 33.77$ & 0.18 \\
\hline ferritin $(\mu \mathrm{g} / \mathrm{L})$ & $30-400$ & 204 & $152.58 \pm 152.67$ & 54 & $140.13 \pm 259.47$ & 0.02 \\
\hline $\mathrm{Tf}(\mathrm{mg} / \mathrm{dL})$ & $200-360$ & 204 & $257.75 \pm 42.62$ & 54 & $261.86 \pm 48.43$ & 0.36 \\
\hline TSAT (\%) & $16-45$ & 204 & $26.84 \pm 11.90$ & 54 & $28.72 \pm 35.34$ & 0.31 \\
\hline sTfR (mg/L) & $<4,5 /<5$ & 78 & $3.59 \pm 3.60$ & 39 & $3.66 \pm 2.34$ & 0.18 \\
\hline ferritin index ${ }^{(1)}$ & $<3.2 /<2$ & 78 & $1.97 \pm 3.18$ & 39 & $2.26 \pm 2.28$ & 0.18 \\
\hline hemoglobin (g/L) & $120-157$ & 204 & $142.80 \pm 16.93$ & 52 & $139.44 \pm 17.22$ & 0.2 \\
\hline $\mathrm{MCH}(\mathrm{pg})$ & $27.0-32.0$ & 204 & $30.65 \pm 2.52$ & 53 & $29.69 \pm 4.43$ & 0.1 \\
\hline $\operatorname{MCV}(\mathrm{fl})$ & $77.0-96.0$ & 204 & $89.50 \pm 6.00$ & 53 & $88.69 \pm 9.48$ & 0.3 \\
\hline $\mathrm{MCHC}(\mathrm{g} / \mathrm{dL})$ & $310-360$ & 204 & $342.32 \pm 13.09$ & 53 & $331.89 \pm 36.83$ & 0.16 \\
\hline CRP (mg/dL) & $0.00-0.50$ & 204 & $0.57 \pm 0.73$ & 53 & $0.85 \pm 1.30$ & 0.42 \\
\hline $\begin{array}{l}\text { creatinine } \\
(\mathrm{mg} / \mathrm{dL})\end{array}$ & $0.67-1.17$ & 203 & $1.06 \pm 0.56$ & 54 & $1.06 \pm 0.40$ & 0.19 \\
\hline
\end{tabular}

Quantitative variables are shown as mean \pm standard deviation; \#parameters are represented as total $\mathrm{n}$ and percentage; (1) ferritin index was calculated as (sTFR/log Ferritin).

\section{Prevalence and characterization of ID and Anemia}

In our study population, 139 patients $(68.1 \%)$ had no ID, while 25 patients $(12.3 \%)$ revealed CID, 21 patients (10.3\%) FID and 19 patients (9.3\%) AID (Figure 3). The absolute number of patients with ID increased during follow-up; however, the observed

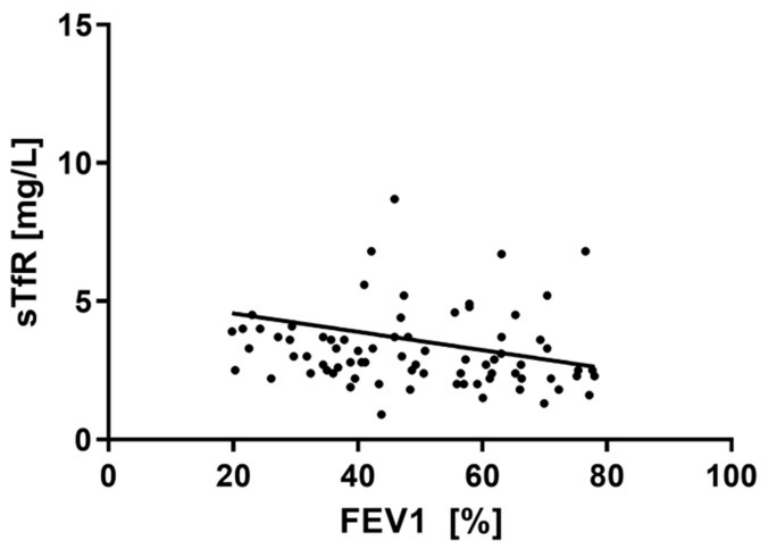

changes between the two time points were not significant $(p>0.05)$ (Figure 3).

Iron-replete patients had a significantly higher DLCOc as compared to patients with ID (63.47 \pm 21.64 vs $50.80 \pm 19.25 \%, p<0.01)$, while CRP $(0.52 \pm 0.68$ vs $0.69 \pm 0.82 \mathrm{mg} / \mathrm{dl}, p=0.03)$ values were significantly lower (Figure 4). There were no significant differences in age, FEV1, CAT-score and LVEF when comparing ID with iron-replete patients.

The mean hemoglobin levels at study inclusion were $142.80 \pm 16.93 \mathrm{~g} / \mathrm{L}$ and showed a slight yet not significant decrease during follow up (139.44 \pm $17.22 \mathrm{~g} / \mathrm{L}, p=0.2)$. Anemia was present in 29 patients $(14.2 \%)$ at study inclusion, and the prevalence increased to $20.8 \%$ during follow-up ( $p=0.5$, Figure 5). Figure 5 shows the distribution of anemia types, IDA, IDA + AI and anemia not related to ID at baseline and follow-up. Patients with anemia had a significantly lower FEV1 (43.19 \pm 17.57 vs $51.49 \pm 17.09 \%, p=0.03$ ) and DLCOc ( $48.35 \pm 17.74$ vs $61.12 \pm 21.78 \%, p=0.01)$, as well as higher CRP levels $(0.77 \pm 0.55$ vs $0.54 \pm 0.75$ $\mathrm{mg} / \mathrm{dl}, p<0.01)$ compared to subjects without anemia irrespective of the presence of ID. CRP levels correlated with ferritin $(r=0.02, p<0.01)$, Tf $(r=-0.220$, $p<0.01)$, and TSAT $(r=-0.281, p<0.001)$.

A total of 116 patients $(56.95 \%)$ received an ACT. No significant differences in $\mathrm{Hb}$, serum iron, ferritin, Tf and TSAT were observed between patients having ACT compared to patients without ACT, whereas $\operatorname{sTfR}(3.70 \pm 2.58$ vs. $3.46 \pm 0.95 \mathrm{mg} / \mathrm{L}, p=0.02)$ and ferritin-index $(2.23 \pm 2.52$ vs. $2.00 \pm 0.94, p<0.01)$ were slightly, yet significantly higher in patients with ACT. Further, the presence of anemia did not show a significant association with ACT $(p=0.42)$ either. A total of $54(26.5 \%)$ of patients had a gastrointestinal comorbidity. No significant differences in $\mathrm{Hb}$, serum iron, Tf, TSAT or sTfR were detected between patients with or without a gastrointestinal condition.

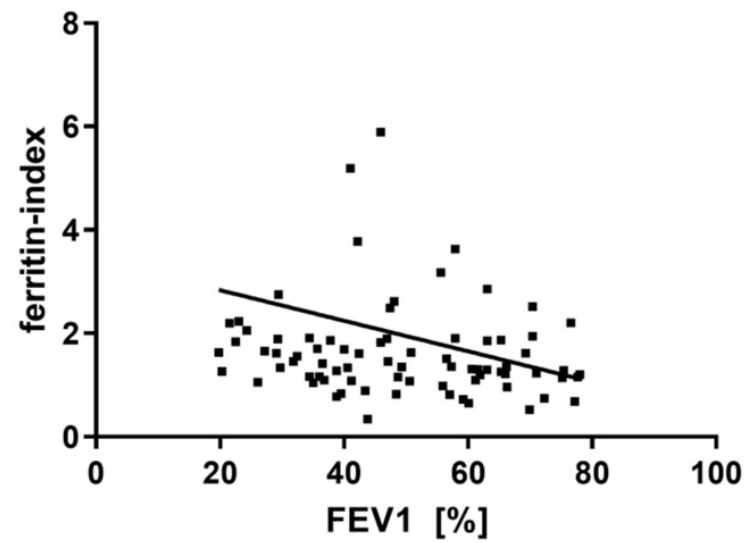

Figure 2. Correlation between FEV1(\%) and sTfR ferritin-index. 


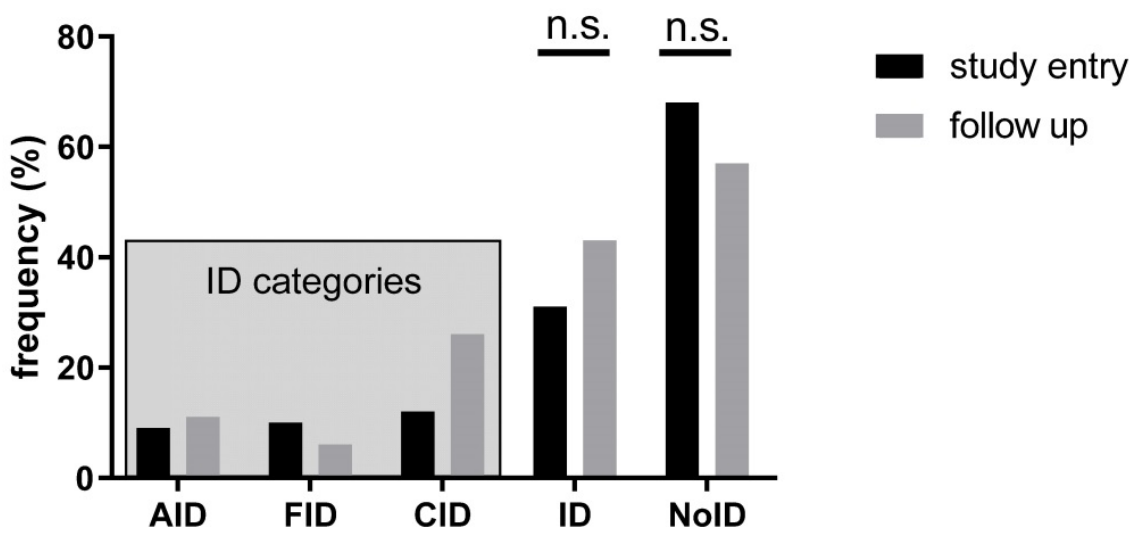

Figure 3. Prevalence of ID at study inclusion and follow-up. AID: absolute iron deficiency, FID: functional iron deficiency, CID: combined iron deficiency, ID: iron deficiency, NolD: no iron deficiency, n.s.: not significant at $p>0.05$.
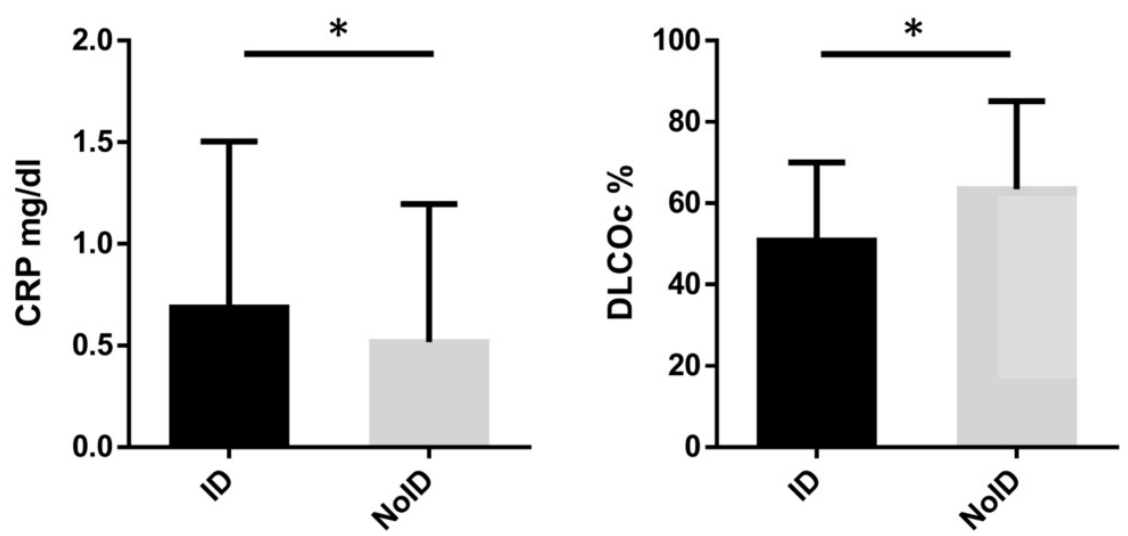

Figure 4. Diffusion capacity and CRP levels depending on iron status. * Indicates statistical significance at $p<0.05$. ID: iron deficiency, NolD: no iron deficiency, DLCOc: diffusion capacity, CRP: C-reactive protein.

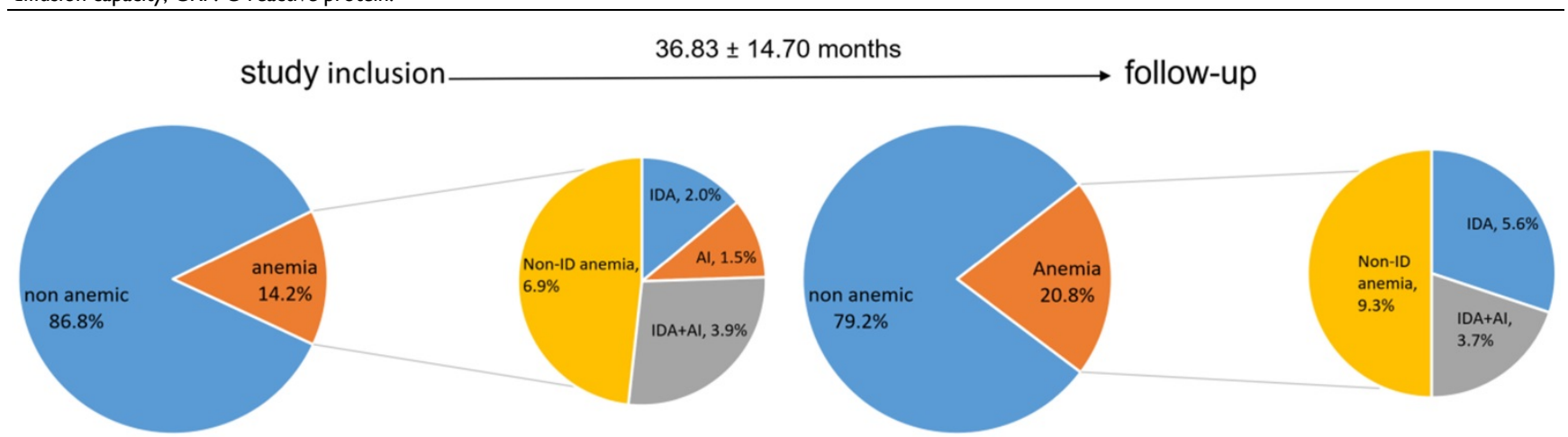

Figure 5. Distribution of anemia, IDA and ACD at study inclusion, and follow-up. IDA: iron deficient anemia, ACD: anemia of chronic disease, ID: iron deficiency.

\section{Discussion}

This study reveals a high prevalence of ID in a real-life COPD cohort, but exact characterization depends on the applied definition. ID is associated with anemia, an elevated inflammatory state, and impaired functional lung capacity, as reflected by FEV1 and DLCOc.

The present study tried to categorize different types of ID, according to commonly applied, robust and easily reproducible ID-definitions. The combined prevalence of all types of ID detected in this study was $30 \%$, the most frequent type being CID. A few studies exploring the possible causality between COPD pathogenesis and severity to ID and/or anemia have already been published [21-27], but the comparison of ID frequencies is difficult as definitions vary across the available literature. Nickol et al. were the first ones to investigate ID using definitions targeting absolute ID and identifying $18 \%$ to be iron-deficient [21], which is distinctly more than $9.3 \%$ of AID in our cohort. FID was previously described in small observational studies using more liberal definitions, therefore not allowing a direct comparison of prevalence rates [25, 28]. The unambiguous definition of ID is crucial, as 
therapeutic supplementation could prove beneficial only in selected patients. Anemic patients and those with established AID benefit from iron supplementation. In FID, on the other hand, its therapeutic effect may be less, as the route of the problem is elevated hepcidin levels. Iron supplementation will most likely not improve iron homeostasis due to the chronic inflammatory state upregulating endogenous hepcidin production [29].

Further, this study is the first to elucidate the course of ID and anemia in COPD over a follow-up period. The observed significant decrease in ferritin and increase of combined ID are mainly driven by chronic inflammatory processes along with blood losses [15-22]. Thus, ID is frequent in COPD and frequently aggravates during the disease. However, as there is no consensus on the exact definition of ID in literature, its estimated prevalence in COPD appears highly variable.

Anemia was present in $14 \%$ of patients at study inclusion, and the prevalence slightly increased during follow-up. Anemia was associated with elevated CRP and a lower FEV1, parameters reflecting a more advanced disease, whereas ACT or gastrointestinal comorbidities did not correlate with anemia. This underlines the current evidence that also anemia is highly prevalent among COPD patients, ranging anywhere between 4.9 and $38.0 \%$ [22, 30, 31]. Anemia of chronic disease is the most common type of anemia in COPD [32] described in the literature, equivalent to this study's findings. The small patient numbers in both studies limit the validity of these results, though. In addition, as ACT did not affect hemoglobin count, its use may be considered safe in this study cohort. Contrasting, a multicenter study of patients with atrial fibrillation, of whom $70 \%$ received an ACT, comparing clinical outcomes in subgroups with and without comorbid COPD revealed a higher incidence of hemorrhagic events in COPD patients [33]. Concerning the relationship between iron parameters and COPD, only sTfR and ferritin-index were negatively associated with FEV1. Parameters like sTfR or ferritin-index allow more accurate characterization of ID as cellular iron demand, iron storage, and systemic inflammation are taken into consideration. COPD stages, the frequency of severe exacerbations, or symptoms assessed as CAT-Score were not related to systemic ID, which is surprising and questions the role of elevated ID prevalence in the pathogenesis of COPD. In this context, it is essential to distinguish between local and systemic iron dyshomeostasis. Previous studies showed high iron content in lung tissues of advanced COPD patients compared to healthy controls, with a heterogeneous distribution of iron at a cellular level, including mitochondria [34]. Mitochondrial iron loading has previously been linked to COPD, as it is upregulated by iron-responsive element-binding protein 2 , which is increased in COPD. Its deficiency seems to alleviate cigarette-smoke induced pulmonary inflammation [35] because mitochondrial iron loading impairs mitochondrial function and oxidative phosphorylation [36]. Moreover, Yoshida et al. showed that cigarette smoke exposure induces ferroptosis, an iron-dependent form of regulated cell death, which may play a role in COPD pathogenesis [9]. Therefore, unbiased correction of ID of any cause may improve systemic iron homeostasis but could have undesired different loco-regional effects in the lung [11]. This study highlights that randomized prospective trials investigating the benefit of iron supplementation in COPD are warranted to improve the clinical management of these patients.

Although our data expand the current knowledge about ID in COPD patients, we have to acknowledge some limitations, the major one being the retrospective design. Thus, the observation period was mainly based on the availability of laboratory studies. Also, about $2 / 3$ of the patients were lost during follow-up, biasing the results, especially in terms of anemia, given the low prevalence, especially in absolute numbers. Further, the potential role of nutrition and pulmonary cachexia contributing to ID could not be addressed due to the retrospective study design. Further prospective studies, including organ-specific iron assessment as well as interventional studies investigating the benefit of iron supplementation, are warranted to confirm the herein presented hypotheses.

\section{Conclusion}

ID is common among COPD patients, but the prevalence highly depends on the definition applied, and a uniform definition for accurate diagnosis does not exist. ID is most frequently caused by a combination of absolute and functional ID, and prevalence is increasing during the course of the disease. ID associates with FEV1 and DLCOc restriction, especially when using more robust parameters like sTfR and ferritin-index, which account for inflammation. Respiratory physicians need to be aware of the high prevalence, as it might reflect a more severe COPD phenotype. Prior to iron supplementation, the underlying type of ID should be identified and especially with the presence of AID extended screening to rule out occult blood losses needs to be considered. Proper detection and treatment options of ID need to be evaluated in future prospective studies. 


\section{Abbreviations}

ACT: Anticoagulation therapy; AI: anemia of inflammation; AID: absolute iron deficiency; CAT: COPD assessment test; CID: combined iron deficiency; COPD: chronic obstructive pulmonary disease; DLCOc: diffusion capacity for carbon monoxide; FEV1: forced expiratory volume in one second; FID: functional iron deficiency; FVC: forced vital capacity; GOLD: Global Initiative for Chronic Obstructive Lung Diseas; ID: iron deficiency; IDA: iron deficiency anemia; LVEF: left ventricular ejection fraction; sTfR: soluble transferrin receptor.

\section{Acknowledgments}

We acknowledge Doris Hüttenberger and Graziella Plank for supporting this project.

\section{Ethics approval}

All procedures performed in the present study involving human participants were in accordance with the ethical standards of the Institutional and/or National Research Committee and with the 1964 Helsinki declaration and its later amendments and were performed after approval of the Ethics Committee of the Medical University of Innsbruck (EK Nr.1147/2017).

\section{Availability of data and materials}

All relevant data are within the paper. The data used to support the findings of this study are available from the corresponding author upon request (Judith.Loeffler@i-med.ac.at, guenter.weiss@i-med.ac. at). Data cannot be shared publicly because of privacy concerns.

\section{Funding}

This work was supported by the Austrian National bank Fund (Project 17271, J.LR.), by the Verein zur Förderung von Forschung und Weiterbildung in Infektiologie und Immunologie, Innsbruck, Austria (G.W.) and the Christian Doppler Society, Austria (G.W.).

\section{Authors' contributions}

Alex Pizzini: wrote the manuscript, contributed to the conception and design of the study, the acquisition of data and the analysis and interpretation.

Magdalena Aichner: contributed to the conception and design of the study, the acquisition of data and the analysis and interpretation and co-wrote the paper.

Thomas Sonnweber: study design, data analysis and interpretation.

Ivan Tancevski: conception and design of the study.

Günter Weiss: conception and design of the study, data analysis and interpretation, cocorresponding author.

Judith Löffler-Ragg: conception and design of the study, data analysis and interpretation, corresponding author.

\section{Competing Interests}

The authors have declared that no competing interest exists.

\section{References}

1. WHO. The top 10 causes of death: WHO; 2018 [updated 25.05.2018. Available from:

https://www.who.int/news-room/fact-sheets/detail/the-top-10-causes-of-d eath.

2. Kirkham PA, Barnes PJ. Oxidative stress in COPD. Chest. 2013; 144(1): 266-73.

3. Fischer BM, Pavlisko E, Voynow JA. Pathogenic triad in COPD: oxidative stress, protease-antiprotease imbalance, and inflammation. Int $\mathrm{J}$ Chron Obstruct Pulmon Dis. 2011; 6: 413-21.

4. Zinellu E, Zinellu A, Fois AG, Carru C, Pirina P. Circulating biomarkers of oxidative stress in chronic obstructive pulmonary disease: a systematic review. Respir Res. 2016; 17(1): 150.

5. Volani C, Paglia G, Smarason SV, Pramstaller PP, Demetz E, Pfeifhofer-Obermair C, et al. Metabolic Signature of Dietary Iron Overload in a Mouse Model. Cells. 2018; 7(12)

6. Muckenthaler MU, Rivella S, Hentze MW, Galy B. A Red Carpet for Iron Metabolism. Cell. 2017; 168(3): 344-61.

7. Ghio AJ. Disruption of iron homeostasis and lung disease. Biochim Biophys Acta. 2009; 1790(7): 731-9

8. DeMeo DL, Mariani T, Bhattacharya S, Srisuma S, Lange C, Litonjua A, et al. Integration of genomic and genetic approaches implicates IREB2 as a COPD susceptibility gene. Am J Hum Genet. 2009; 85(4): 493-502.

9. Yoshida M, Minagawa S, Araya J, Sakamoto T, Hara H, Tsubouchi K, et al. Involvement of cigarette smoke-induced epithelial cell ferroptosis in COPD pathogenesis. Nat Commun. 2019; 10(1): 3145.

10. Chung KF. Cytokines in chronic obstructive pulmonary disease. Eur Respir J Suppl. 2001; 34: 50s-9s.

11. Weiss G, Ganz T, Goodnough LT. Anemia of inflammation. Blood. 2019;133(1):40-50.

12. Camaschella C. Iron deficiency. Blood. 2019; 133(1): 30-9.

13. Weiss G, Goodnough LT. Anemia of chronic disease. The New England journal of medicine. 2005; 352(10): 1011-23.

14. Punnonen K, Suominen P, Kuusinen A, Kuiper-Kramer E. Clinical use of soluble transferrin receptor. Clinical chemistry and laboratory medicine. 2000; 38(4): 377

15. Brugnara C. Iron deficiency and erythropoiesis: new diagnostic approaches. Clinical chemistry. 2003; 49(10): 1573-8.

16. Thomas L, Thomas C. Detection of iron restriction in anaemic and non-anaemic patients: New diagnostic approaches. European journal of haematology. 2017; 99(3): 262-8.

17. Punnonen K, Irjala K, Rajamaki A. Serum transferrin receptor and its ratio to serum ferritin in the diagnosis of iron deficiency. Blood. 1997; 89(3): 1052-7.

18. Weiss G. Anemia of Chronic Disorders: New Diagnostic Tools and New Treatment Strategies. Seminars in hematology. 2015; 52(4): 313-20.

19. Singh D, Agusti A, Anzueto A, Barnes PJ, Bourbeau J, Celli BR, et al. Global Strategy for the Diagnosis, Management, and Prevention of Chronic Obstructive Lung Disease: the GOLD science committee report 2019. The European respiratory journal. 2019; 53(5).

20. Greiner S, Jud A, Aurich M, Hess A, Hilbel T, Hardt S, et al. Reliability of noninvasive assessment of systolic pulmonary artery pressure by Doppler echocardiography compared to right heart catheterization: analysis in a large patient population. J Am Heart Assoc. 2014; 3(4)

21. Nickol AH, Frise MC, Cheng HY, McGahey A, McFadyen BM, Harris-Wright $\mathrm{T}$, et al. A cross-sectional study of the prevalence and associations of iron deficiency in a cohort of patients with chronic obstructive pulmonary disease. BMJ open. 2015;5(7):e007911. doi: 10.1136/bmjopen-2015-007911.

22. Robalo Nunes A, Tátá M. The impact of anaemia and iron deficiency in chronic obstructive pulmonary disease: A clinical overview. Rev Port Pneumol (2006). 2017; 23(3): 146-55.

23. Hoepers AT, Menezes MM, Fröde TS. Systematic review of anaemia and inflammatory markers in chronic obstructive pulmonary disease. Clin Exp Pharmacol Physiol. 2015; 42(3): 231-9.

24. Horadagoda C, Dinihan T, Roberts M, Kairaitis K. Body composition and micronutrient deficiencies in patients with an acute exacerbation of chronic obstructive pulmonary disease. Intern Med J. 2017. 
25. Barberan-Garcia A, Rodríguez DA, Blanco I, Gea J, Torralba Y, Arbillaga-Etxarri A, et al. Non-anaemic iron deficiency impairs response to pulmonary rehabilitation in COPD. Respirology. 2015; 20(7): 1089-95.

26. Kentson $M$, Leanderson $\mathrm{P}$, Jacobson $\mathrm{P}$, Persson HL. Oxidant status, iron homeostasis, and carotenoid levels of COPD patients with advanced disease and LTOT. Eur Clin Respir J. 2018; 5(1): 1447221. doi: 10.1080/20018525.2018.1447221. [eCollection 2018].

27. Plesner LL, Schoos MM, Dalsgaard M, Goetze JP, Kjoller E, Vestbo J, et al. Iron Deficiency in COPD Associates with Increased Pulmonary Artery Pressure Estimated by Echocardiography. Heart Lung Circ. 2017; 26(1): 101-4.

28. Plesner LL, Schoos MM, Dalsgaard M, Goetze JP, Kjøller E, Vestbo J, et al. Iron Deficiency in COPD Associates with Increased Pulmonary Artery Pressure Estimated by Echocardiography. Heart Lung Circ. 2017; 26(1): 101-4.

29. Cloonan SM, Mumby S, Adcock IM, Choi AMK, Chung KF, Quinlan GJ. The "Iron"-y of Iron Overload and Iron Deficiency in Chronic Obstructive Pulmonary Disease. Am J Respir Crit Care Med. 2017; 196(9): 1103-12.

30. Fraenkel PG. Understanding anemia of chronic disease. Hematology Am Soc Hematol Educ Program. 2015; 2015: 14-8.

31. Boutou A, Hopkinson N, Polkey M. Anaemia in chronic obstructive pulmonary disease : an insight into its prevalence and pathophysiology. 2015; 128(5): 283-95.

32. Schneckenpointner R, Jörres RA, Meidenbauer N, Kollert F, Pfeifer M, Budweiser S. The clinical significance of anaemia and disturbed iron homeostasis in chronic respiratory failure. Int J Clin Pract. 2014; 68(1): 130-8.

33. Rodríguez-Mañero M, López-Pardo E, Cordero A, Ruano-Ravina A, Novo-Platas J, Pereira-Vázquez M, et al. A prospective study of the clinical outcomes and prognosis associated with comorbid COPD in the atrial fibrillation population. International journal of chronic obstructive pulmonary disease. 2019; 14: 371-80.

34. Najafinobar N, Venkatesan S, von Sydow L, Klarqvist M, Olsson H, Zhou XH, et al. ToF-SIMS mediated analysis of human lung tissue reveals increased iron deposition in COPD (GOLD IV) patients. Sci Rep. 2019; 9(1): 10060

35. Cloonan SM, Glass K, Laucho-Contreras ME, Bhashyam AR, Cervo M, Pabón MA, et al. Mitochondrial iron chelation ameliorates cigarette smoke-induced bronchitis and emphysema in mice. Nat Med. 2016; 22(2): 163-74.

36. Volani C, Doerrier C, Demetz E, Haschka D, Paglia G, Lavdas AA, et al. Dietary iron loading negatively affects liver mitochondrial function. Metallomics : integrated biometal science. 2017; 9(11): 1634-44 